\title{
A nonlinear Klein-Gordon equation for relativistic superfluidity
}

\author{
Oliver Waldron and Robert A. Van Gorder* \\ Mathematical Institute, University of Oxford \\ Andrew Wiles Building, Radcliffe Observatory Quarter, Woodstock Road, Oxford, OX2 6GG, United Kingdom \\ Email: Robert.VanGorder@maths.ox.ac.uk
}

August 3, 2017

\begin{abstract}
Many neutron star features can be accurately modeled only if one assumes that a significant portion of the neutron star interior is in a superfluid state and if relativitic effects are considered, and possible solutions to the underlying mathematical models include vortex solutions. It was recently shown that vorticity in relativistic superfluids can be studied under the framework of a nonlinear Klein-Gordon (NLKG) model in general curvilinear coordinates where the phase dynamics of solutions to this equation give rise to superfluidity [Xiong et al., Physical Review D 90 (2014) 125019], and some numerical solutions were obtained. The aim of this paper will be to extract asymptotic solutions to obtain a better qualitative understanding of the possible relativistic superfluid dynamics possible under the NLKG model. We obtain asymptotic results for both spherically symmetric and cylindrically symmetric solutions, demonstrating that the solutions actually appear more regular in the relativistic regime compared to the non-relativistic limit. In fact, the asymptotic and numerical solutions actually show the best agreement in the relativistic case. We demonstrate that the relativistic effects actually tend to regularize or stabilize the solutions, relative to the non-relativistic solutions, which is an interesting finding. We then obtain a Thomas-Fermi-like perturbation result in the very large-mass limit where the kinetics become negligible relative to the self-interaction term (at leading order). We finally extend the NLKG model by assuming a curved spacetime with a metric generally used to model the space surrounding a neutron star, which is a novel generalization of the NLKG model to curved spacetime. We again obtain solutions in the large-mass limit for this case, and find that for such a spacetime non-stationary states (rather than simply stationary states) are possible in the large-mass limit.
\end{abstract}

Keywords: relativistic superfluidity; nonlinear Klein-Gordon equation; neutron stars

\section{Introduction}

Motivated by observational data on neutron stars from pulsar timing measurements, it was found that many neutron star features could be accurately modeled only if one assumes that a significant portion of the neutron star interior is in a superfluid state [1], and this motivated a number of theoretical studies on such structural configurations. Such models of the dynamics of the neutron superfluid in neutron stars include $[1,2]$. A model for the dynamical coupling of the neutron star superfluid core is based on the dynamics of a neutron vortex with a strong magnetic field was also considered in [3]. Importantly, relativistic corrections to the Newtonian theory for neutron stars 
are of the order of 20-30 per cent [1], necessitating the inclusion of relativistic terms in numerical simulations $[4,5]$. A Hamiltonian formulation for general relativistic superfluids was considered in [6]. These studies suggest that, in order to properly model and understand the dynamics within the interior of neutron stars, further study of the dynamics of relativistic superfluids is needed.

One very interesting feature seen in relativistic simulations of neutron star cores is the emergence of vortex solutions. Vortex rings were considered in [7], where they emerge as stationary solutions to the governing equations. Their stability was studied, and the dynamics of the vortex ring solutions was related back to smoke rings in an inviscid fluid. Vortex solutions have also been discussed in the context of strings in the relativistic setting $[8,9]$. Vortices in the relativistic Gross-Pitaevskii (GP) equation have been studied [10], while superconducting-superfluid mixtures have also been shown to admit vorticies in the relativistic case [11].

There is quite a literature available on vortex dynamics in cold superfluids, such as superfluid helium. It is common to study the dynamics of quantised vortex filaments in superfluids (such as superfluid Helium) under the local induction approximation (LIA) [12]. The LIA can be put into correspondence with a cubic non-linear Schrödinger (NLS) equation [13], and this is helpful for obtaining solutions describing nonlinear waves, such as solitons, along quantised vortex filaments [14]. Superfluidity is found to arise as a consequence of macroscopic phase coherence. That is, cases where the quantum phase of a state correlates over macroscopic distances [15]. The situations in which this may occur are many and diverse, as detailed in [15]. Among these scenarios are liquid ${ }^{4} \mathrm{He}$ at temperatures near absolute zero, Higgs fields over cosmological scales, and superconductivity on the interior of a neutron star, which may be viewed as superfluidity of condensed fermion pairs $[15]$.

We begin our discussion with a review of the progress made by Xiong et al. [15], by describing the phenomenology of a superfluid in curved spacetime in terms of a complex scalar field $\Phi$ satisfying a nonlinear Klein-Gordon equation (NLKG) of the form

$$
\square \Phi+f(\Phi)=0,
$$

where $\square$ generalises the operator $\partial^{\mu} \partial_{\mu}=\nabla^{2}-\frac{1}{c^{2}} \frac{\partial^{2}}{\partial t^{2}}$ to curvilinear coordinates. This phenomenological model was initially proposed by Ginzburg and Landau ([15, 16]), with the aim of modeling superfluidity as a manifestation of macroscopic phase coherence in quantum systems. This phase coherence usually occurs in Bose-Einstein Condensates $([15,17])$, which arise in a diverse range of physical situations [15]. As a point of physical interest, [15] places the field $\Phi$ in the form

$$
\Phi=F e^{i \sigma},
$$

and identifies the superfluid velocity as being proportional to the gradient $\nabla \sigma$. We also identify in [15] a Coriolis and centrifugal force defined by

$$
R_{\text {coriolis }}=\frac{2 \Omega}{c^{2}} \frac{\partial^{2}}{\partial \phi \partial t} \quad \text { and } \quad R_{\text {centrifugal }}=-\frac{\Omega^{2}}{c^{2}} \frac{\partial^{2}}{\partial \phi^{2}}
$$

where Xiong et al. identify $\Omega$ as an angular velocity, and $\phi$ is the angle of rotation [15].

In [15], Xiong et al. proceed to pick a particular form for the nonlinear potential, $f(\Phi)=$ $-V^{\prime}\left(\Phi^{*} \Phi\right) \Phi$, where $V$ Is a general self-interacting potential. This gives equation (1) the form

$$
\left(\partial^{\mu} \partial_{\mu}-V^{\prime}\right) \Phi=0 .
$$


From here, [15] defines a wave function $\Psi$ through the substitution

$$
\Phi(\mathbf{x}, t)=e^{-i \omega_{0} t} \Psi(\mathbf{x}, t)
$$

and a mass scale $m$ through defining $\omega_{0}=\frac{m c^{2}}{\hbar}$. This mass scale relates the magnitude of $\Psi$ to the superfluid density through the relation $\rho_{s}=m|\Psi|^{2}$ [15]. Plugging this substitution into equation (3) gives a relativistic extension of the Nonlinear Schrödinger equation (NLSE):

$$
i \hbar \frac{\partial \Psi}{\partial t}=-\frac{\hbar^{2}}{2 m} \nabla^{2} \Psi+\frac{\hbar^{2}}{2 m c^{2}} \frac{\partial^{2} \Psi}{\partial t^{2}}+U\left(|\Psi|^{2}\right) \Psi,
$$

where we have defined a modified potential,

$$
U=\frac{\hbar^{2}}{2 m} V^{\prime}-\frac{m c^{2}}{2}
$$

in order to collect the terms multiplying $\Psi$. In this formulation, the rotational forces will have a slightly different affect on the field:

$$
R_{\text {coriolis }}(\Phi)=R_{\text {coriolis }}\left(e^{-i \omega_{0} t} \Psi\right)=-\frac{2 \Omega e^{-i \omega_{0} t}}{c^{2}}\left(\frac{\partial^{2} \Psi}{\partial t \partial \phi}-i \omega_{0} \frac{\partial \Psi}{\partial \phi}\right)
$$

and

$$
R_{\text {centrifugal }}(\Phi)=R_{\text {centrifugal }}\left(e^{-i \omega_{0} t} \Psi\right)=-\frac{\Omega^{2} e^{-i \omega_{0} t}}{c^{2}} \frac{\partial^{2} \Psi}{\partial \phi^{2}} .
$$

This leads to altered definitions of the terms:

$$
\begin{gathered}
R_{\text {coriolis }}^{(S)}=-\frac{2 \Omega e^{-i \omega_{0} t}}{c^{2}}\left(\frac{\partial^{2}}{\partial t \partial \phi}-i \omega_{0} \frac{\partial}{\partial \phi}\right), \\
R_{\text {centrifugal }}^{(S)}=-\frac{\Omega^{2} e^{-i \omega_{0} t}}{c^{2}} \frac{\partial^{2}}{\partial \phi^{2}} .
\end{gathered}
$$

Xiong et al. [15] then make a number of qualitative deductions from this NLSE formulation, which can be found in [15], before returning to the NLKG in order to make a series of numerical computations. In this paper, however, we will endeavor to obtain asymptotic solutions for a variety of important special cases.

Motivated by the existing numerical results of Xiong et al. [15] on the relativistic NLKG equation, in this paper we shall obtain asymptotic solutions to this relativistic NLKG equation, in order to better understand the qualitaitve features of the solutions. In Section 2, we will consider time dependence as only a phase factor, on order to discuss stationary solutions. We will also pick a form for the potential consistent with a cubic Nonlinear Schrödinger equation, for sake of comparison with the non-relativistic limit. Solutions with spherical or cylindrical symmetry are considered, as these geometries permit solutions. In Section 3, we will treat mass as a large parameter (so that the reciprocal of mass is a small parameter) obtain asymptotic solutions akin to the Thomas-Fermi approximation. In Section 4, will extend the NLKG model considered in the literature by considering the NLKG in a curved spacetime metric generally applied to rotating neutron stars. We will determine the coefficients in the metric, before introducing a mass scale, and considering the limit as that mass scale becomes large. This will again allow us to obtain asymptotic solutions in the large-mass limit. Concluding remarks are given in Section 5. 


\section{Stationary Solutions}

One standard assumption when attempting to solve NLKG equations is to assume a stationary solution. To this effect, we will begin by making the substitution

$$
\Psi(\mathbf{x}, t)=e^{-i \lambda t} \psi(\mathbf{x})
$$

and we say that any complex scalar field $\Psi$ satisfying (5) and (7) is a stationary solution to (5). When we plug this complex scalar field (7) back into our NLSE (5), we obtain a nonlinear Helmholtz equation of the following form:

$$
\nabla^{2} \psi+k^{2} \psi=\hat{U}\left(|\psi|^{2}\right) \psi
$$

where

$$
\hat{U}\left(|\psi|^{2}\right)=\frac{2 m}{\hbar^{2}} U\left(|\psi|^{2}\right)=V^{\prime}\left(|\psi|^{2}\right)-\frac{m^{2} c^{2}}{\hbar^{2}},
$$

and we define $k^{2}=\frac{2 m \lambda}{\hbar}+\frac{\lambda^{2}}{c^{2}}$. When performing numerical solutions in later sections, we pick units so that $m / \hbar=1$.

At this point, we will choose the potential $V$ to satisfy

$$
V^{\prime}\left(|\psi|^{2}\right)=\left(\frac{m c}{\hbar}\right)^{2}+\varepsilon|\psi|^{2}
$$

as per [15]. We have a nonlinear Helmholtz equation [18] of the form

$$
\nabla^{2} \psi+k^{2} \psi=\varepsilon \psi|\psi|^{2} .
$$

Note that we could have chosen $V^{\prime}$ to include higher-order terms. Our choice of $V^{\prime}$ is consistent with the cubic NLSE in the nonrelativistic limit. Even in the case where $\varepsilon$ is not small, we will recover the NLKG equation with cubic nonlinearity.

\subsection{Stationary Solutions with Spherical Symmetry}

To reduce the equation to the simplest case possible, we will convert to polar coordinates, and attempt to eliminate the angular variables using a similar substitution to the one we used to eliminate time dependence:

$$
\psi(r, \theta, \phi)=e^{-i \theta_{0} \theta-i \phi_{0} \phi} R(r),
$$

where $\theta_{0}$ and $\phi_{0}$ are (for now) general constants, and $R(r)$ is real. Expanding the Laplace operator in equation (9) and using our substitution (10), we find the equation

$$
R^{\prime \prime}(r)+\frac{2}{r} R^{\prime}(r)-\frac{i \cot (\theta) \theta_{0}}{r^{2}} R(r)-\frac{\theta_{0}^{2}}{r^{2}} R(r)-\frac{\phi_{0}^{2}}{r^{2} \sin ^{2}(\theta)} R(r)+k^{2} R(r)=\varepsilon R(r)^{3},
$$

where prime denotes differentiation with respect to $r$.However, since the terms in $\cot (\theta)$ and $\operatorname{cosec}^{2}(\theta)$ are linearly independent from each other and from the rest of the equation, we require their coefficients to vanish. This requires setting both $\theta_{0}$ and $\phi_{0}$ to 0 . We are left with $\psi(r, \theta, \phi)=R(r)$, with $R(r)$ satisfying

$$
R^{\prime \prime}(r)+\frac{2}{r} R^{\prime}(r)+k^{2} R(r)=\varepsilon R(r)^{3} .
$$




\subsubsection{Perturbative Solutions}

From this point, we can begin to seek perturbative expansions of $R$ in terms of the nonlinear term on the right-hand side of the above equation.

Assuming $\varepsilon$ is sufficiently small, $R$ will take the form

$$
R(r)=R_{0}(r)+\varepsilon R_{1}(r)+O\left(\varepsilon^{2}\right) .
$$

Plugging this expansion into equation (12), we begin matching up the coefficients of powers of $\varepsilon$. To zeroth order, we obtain the linear ODE

$$
R_{0}^{\prime \prime}(r)+\frac{2}{r} R_{0}^{\prime}(r)+k^{2} R_{0}(r)=0 .
$$

A general solution, which can be checked by substituting into equation (14), takes the form

$$
R_{0}(r)=\frac{1}{k r}(A \sin (k r)+B \cos (k r))
$$

since $k^{2}>0$. Based on physical considerations, we would like for there to be no singularity at the origin, and on this basis, we will set $B=0$ in our solution (15), leaving

$$
R_{0}(r)=\frac{A \sin (k r)}{k r}
$$

Using this result, we are now prepared to solve the inhomogeneous equation for the solution to first order in $\varepsilon$ :

$$
R_{1}^{\prime \prime}(r)+\frac{2}{r} R_{1}^{\prime}(r)+k^{2} R_{1}(r)=R_{0}(r)^{3} .
$$

We attempt to solve this equation using variation of parameters. First, we assume $R_{1}$ takes the form

$$
R_{1}(r)=c_{1}(r) y_{1}(r)+c_{2}(r) y_{2}(r)
$$

where

$$
y_{1}(r)=\frac{\sin (k r)}{k r} \quad \text { and } \quad y_{2}(r)=\frac{\cos (k r)}{k r} .
$$

Using our standard variation of parameter methods, we find that

$$
c_{1}(r)=\int \frac{R_{0}(r)^{3} y_{2}(r)}{w(r)} d r \quad \text { and } \quad c_{2}(r)=-\int \frac{R_{0}(r)^{3} y_{1}(r)}{w(r)} d r,
$$

where $w(r)=y_{1}^{\prime}(r) y_{2}(r)-y_{1}(r) y_{2}^{\prime}(r)$. Evaluating these integrals, and using the boundary conditions $R_{1}(0)=0$ and $R_{1}^{\prime}(0)=0$, we obtain the expression for $R_{1}$ as

$$
R_{1}(r)=\frac{A^{3} \sin (k r)}{2 k^{3} r}(\operatorname{Ci}(2 k r)-\operatorname{Ci}(4 k r)+\ln (2))+\frac{A^{3} \cos (k r)}{2 k^{3} r}(\operatorname{Si}(4 k r)-2 \operatorname{Si}(2 k r)) .
$$

Substituting this expression into (13) gives a first-order solution to the radial stationary state equation, which we can compare to numerical results. 

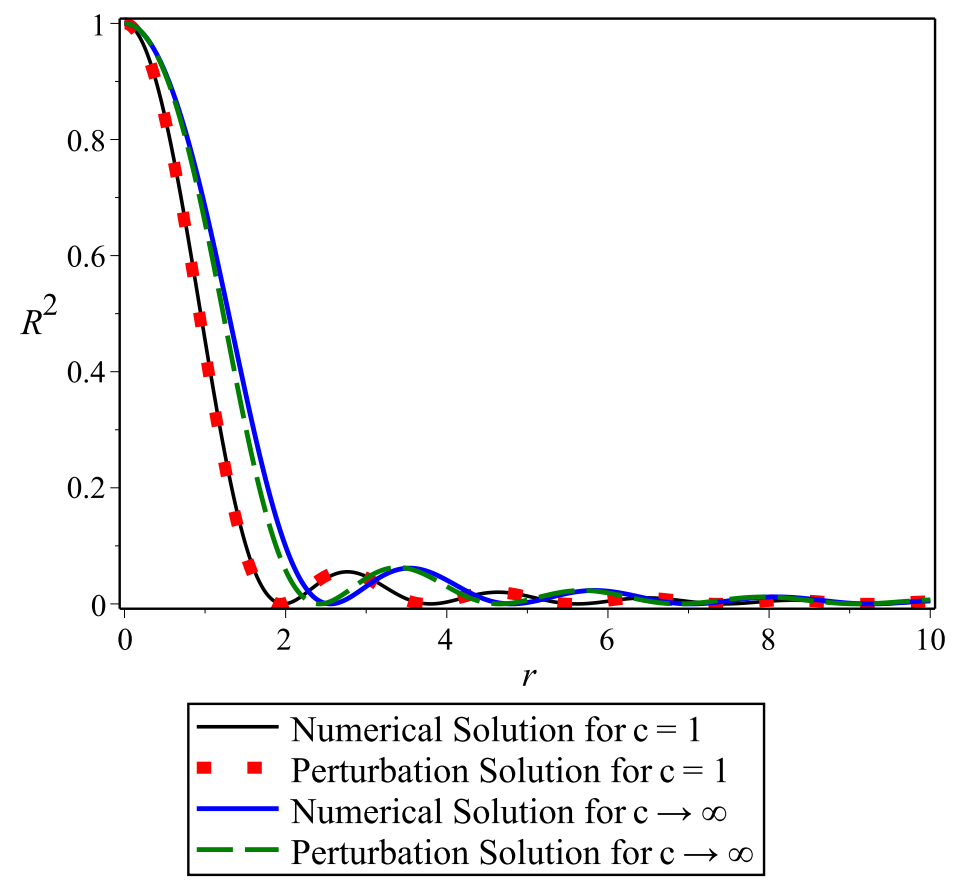

Figure 1: Plot of numerical and perturbative spherically symmetric solutions in the relativistic and nonrelativistic cases, with $\lambda$ and $A$ set equal to 1 , while $\varepsilon=1$.

\subsubsection{Comparison With Numerical Solutions}

We will compare the first-order perturbation solutions obtained in the previous subsection to numerical solutions computed using MatLab's ode45 function, which is based on an explicit Runge-Kutta $(4,5)$ formula.

In Figures 1-3, we plot the perturbative solutions and the numerical solutions in close agreement in both the relativistic $c=1$ case and the nonrelativistic limit $c \rightarrow \infty$, with Figure 1 showing $\varepsilon 1$, Figure 2 showing $\varepsilon=2$ and Figure 3 showing $\varepsilon=2.001$. We retain first-order corrections; that is, we plot $R_{0}(r)+\varepsilon R_{1}(r)$.

When the strength of the nonlinearity is increased, the perturbative solution becomes significantly less accurate, although this lack of accuracy appears to depend on the value of $c$. The numerical solution in the nonrelativistic limit has blown up with the nonlinearity at strength $\varepsilon>2$, suggesting that the solution ceases to be physical with the nonlinearity at this strength. Note the effect that changing the linear coefficient of $R$ in the differential equation has on the accuracy of the perturbation as the nonlinearity grows in strength. The value of $c$ only appears as a denominator in the expression for $k^{2}$, suggesting that for larger $k$, the solution remains accurate for larger strengths of nonlinearity. We see that once $\varepsilon$ reaches a certain critical value, the numerical solution blows up, suggesting that the solution ceases to be physical at this point. For all cases considered, the relativistic asymptotic solution is a very good match (in a qualitative sense) to the numerical simulation.

Figure 4 is given to demonstrate how this critical value of $\varepsilon$ (rounded to the nearest 0.01 ) varies as we vary the value of $c$. The plot is only reliable for relatively small values of $c$, as we are changing 


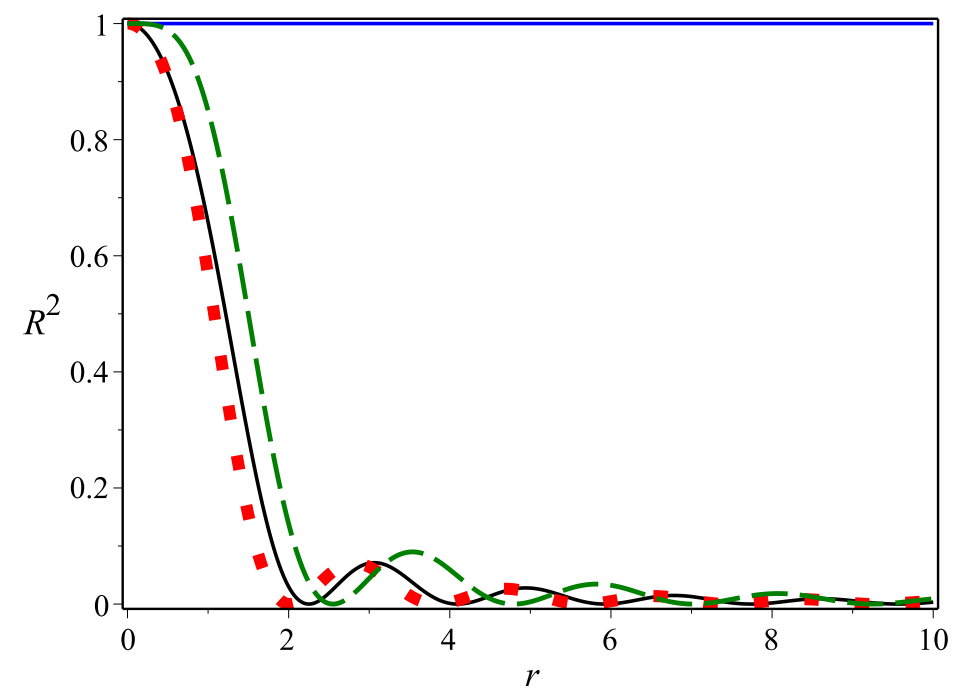

Numerical solution for $\mathrm{c}=1$

- Perturbation Solution for $\mathrm{c}=1$

Numerical Solution for $\mathrm{c} \rightarrow \infty$

-- Perturbation Solution for $\mathrm{c} \rightarrow \infty$

Figure 2: Plot of numerical and perturbative spherically symmetric solutions in the relativistic and nonrelativistic cases, with $\lambda$ and $A$ set equal to 1 , while $\varepsilon=2$.

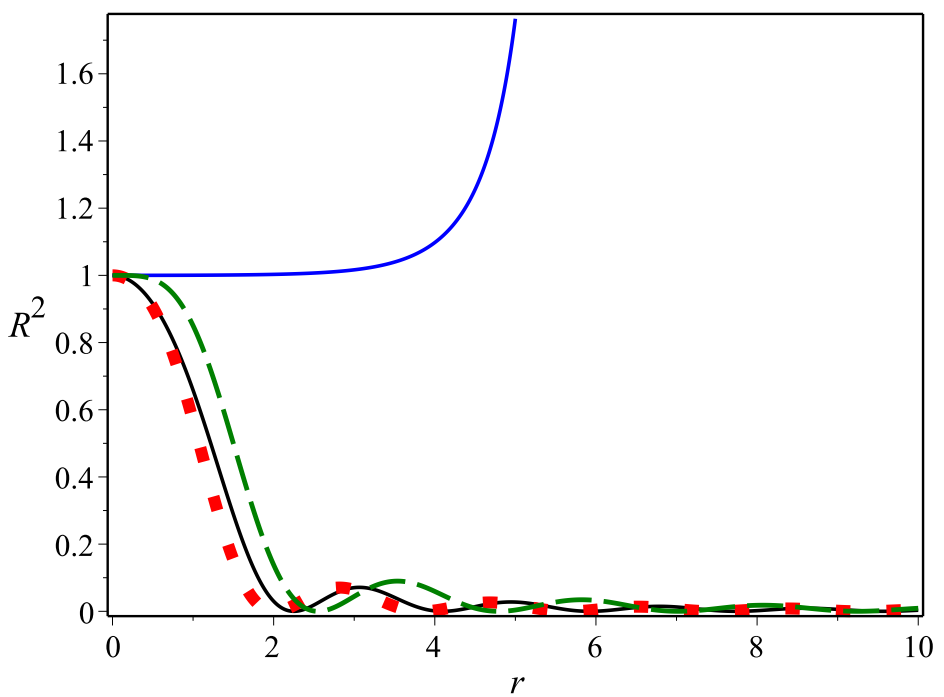

Numerical Solution for $\mathrm{c}=1$

- Perturbation Solution for $\mathrm{c}=1$

Numerical Solution for $\mathrm{c} \rightarrow \infty$

-- Perturbation Solution for $\mathrm{c} \rightarrow \infty$

Figure 3: Plot of numerical and perturbative spherically symmetric solutions in the relativistic and nonrelativistic cases, with $\lambda$ and $A$ set equal to 1 , while $\varepsilon=2.001$. 


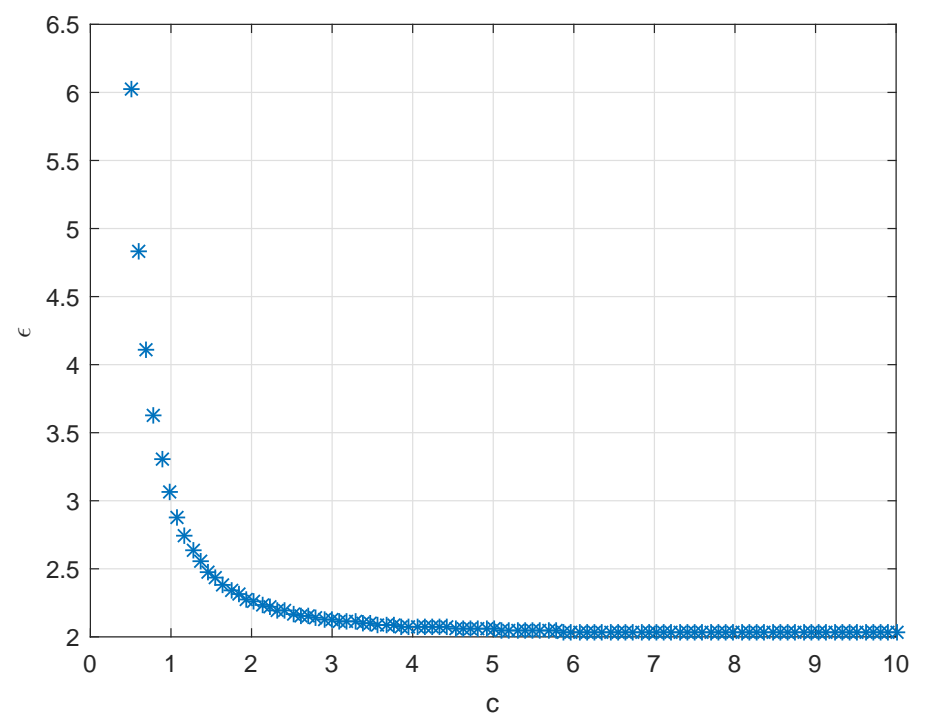

Figure 4: The critical value of $\varepsilon$ rounded to the nearest 0.01 , versus the value of $\mathrm{c}$. Beyond this value of $\varepsilon$, the numerical solutions suggest blowup, implying that the solutions are no longer physical for such strong nonlinearity. Asymptotically, the critical value of the strength of the nonlinearity $\varepsilon$ approaches two as $c$ tends to infinity (the non-relativistic limit).

our units to vary $c$ while neglecting the corresponding effect the change of units should have on $\hbar$. Still, we see that by including small $c$ (under a geometrised unit system, $c=1$ ) the perturbative solutions are better behaved then their non-relativistic $(c \rightarrow \infty)$ counterparts, implying that the relativistic solutions maintain regularity for far stronger nonlinearities than do their non-relativistic counterparts.

Of course, we have two major caveats when considering Figure 4. The shift in the value of $\hbar$ corresponding to the shift in the value of $c$ has not been taken into account, and the fact that the critical value of $\varepsilon$ was rounded means that we cannot see the finer details of convergence from this plot. Nevertheless, it demonstrates the important role that the speed of light plays in these relativistic fluid mechanics, and gives the surprising result that the numerical methods are more reliable in the relativistic regime. We can attribute this to a physical property of the system: the lower the value of $c$, the more restricted we consider communication between individual fluid elements to be. When that restriction is removed completely, it allows solutions to blow up faster because any given fluid element is being instantaneously affected by the rest of the fluid across all of space at once (due to the infinite speed of propagation, $c \rightarrow \infty$ ).

\subsection{D Solutions with Cylindrical Symmetry}

We now consider a different reduction of (9), in which the solutions have cylindrical symmetry. In cylindrical coordinates, we will make the substitution

$$
\psi(r, \phi, z)=e^{-i z_{0} z} P(r, \phi),
$$


with $P$ real. Expanding the Laplacian in equation (9) and using this substitution, we arrive at the equation

$$
\frac{\partial^{2} P}{\partial r^{2}}+\frac{1}{r} \frac{\partial P}{\partial r}+\frac{1}{r^{2}} \frac{\partial^{2} P}{\partial \phi^{2}}+\left(k^{2}-z_{0}^{2}\right) P=\varepsilon P^{3} .
$$

Recall from (2) the definition of the centrifugal operator:

$$
R_{\text {centrifugal }}(\Phi)=-\frac{\Omega^{2}}{c^{2}} \Phi_{\phi \phi}=-\frac{e^{-i\left(\omega_{0} t+\lambda t+z_{0} z\right)} \Omega^{2}}{c^{2}} P_{\phi \phi}
$$

A term of this form appears in (21). We may therefore consider the cylindrically symmetric solution to be a stationary solution in a general rotating frame.

\subsubsection{Perturbative Solutions}

As before, we will expand $P$ in powers of $\varepsilon$,

$$
P(r, \phi)=P_{0}(r, \phi)+\varepsilon P_{1}(r, \phi)+O\left(\varepsilon^{2}\right),
$$

and compare the coefficients of each power of $\varepsilon$.

To zeroth order, we will then obtain the linear equation

$$
\frac{\partial^{2} P_{0}}{\partial r^{2}}+\frac{1}{r} \frac{\partial P_{0}}{\partial r}+\frac{1}{r^{2}} \frac{\partial^{2} P_{0}}{\partial \phi^{2}}+\left(k^{2}-z_{0}^{2}\right) P_{0}=0
$$

This can be solved by separation of variables, with Bessel functions in the radial coordinate and trigonometric functions in the angular coordinate. The full, general expression of $P_{0}$ is then

$$
P_{0}(r, \phi)=\sum_{n=0}^{\infty}\left(A_{n} J_{n}\left(r \sqrt{k^{2}-z_{0}^{2}}\right)+B_{n} Y_{n}\left(r \sqrt{k^{2}-z_{0}^{2}}\right)\right)\left(C_{n} \cos (n \phi)+D_{n} \sin (n \phi)\right) .
$$

Note that for this solution to be stable, we will require that $\left|z_{0}\right|<|k|$. This tells us that a solution with cylindrical symmetry may be periodic in the $z$ coordinate, but the frequency of this periodicity must remain below a certain value in order for the solution to remain stable, and therefore for our model to be physical.

Again, owing to the fact that we wish to avoid dealing with singularities at the origin, we will here set $B_{n}$ to zero for every n, allowing us to absorb $A_{n}$ into $C_{n}$ and $D_{n}$. With a little relabeling, our expression for $P_{0}$ now reads

$$
P_{0}(r, \phi)=\sum_{n=0}^{\infty} J_{n}\left(r \sqrt{k^{2}-z_{0}^{2}}\right)\left(A_{n} \sin (n \phi)+B_{n} \cos (n \phi)\right) .
$$

Now that we have our linear solution, we may begin finding the first-order correction with the nonlinearity included. Consider equation (21), with $P$ expanded in $\varepsilon$ as above. Matching up coefficients of $\varepsilon$, we find an equation for $P_{1}$ :

$$
\frac{\partial^{2} P_{1}}{\partial r^{2}}+\frac{1}{r} \frac{\partial P_{1}}{\partial r}+\frac{1}{r^{2}} \frac{\partial^{2} P_{1}}{\partial \phi^{2}}+\left(k_{\alpha}^{2}-z_{0}\right) P_{1}=P_{0}^{3}
$$


We will solve this using the method of Green's functions. Following the method of contour integration outlined in [19], we retrieve as Green's functions the Hankel functions:

$$
\begin{aligned}
& \frac{i}{4} H_{0}^{(1)}\left(\sqrt{k_{\alpha}^{2}-z_{0}^{2}} r\right)=\frac{i}{4}\left[J_{0}\left(\sqrt{k_{\alpha}^{2}-z_{0}^{2}} r\right)+i Y_{0}\left(\sqrt{k_{\alpha}^{2}-z_{0}^{2}} r\right)\right], \\
& \frac{i}{4} H_{0}^{(2)}\left(\sqrt{k_{\alpha}^{2}-z_{0}^{2}} r\right)=\frac{i}{4}\left[J_{0}\left(\sqrt{k_{\alpha}^{2}-z_{0}^{2}} r\right)-i Y_{0}\left(\sqrt{k_{\alpha}^{2}-z_{0}^{2}} r\right)\right] .
\end{aligned}
$$

References [19] and [20] then impose the radiation condition, which states that the solution we desire should act as a wave moving away from the source. That is, it should act as a source and not as a sink. We will impose the same condition, and therefore disregard the second Hankel function. Our Green's function then has the form

$$
G(\mathbf{x} \mid \hat{\mathbf{x}})=\frac{i}{4} H_{0}^{(1)}\left(\sqrt{k_{\alpha}^{2}-z_{0}^{2}}|\mathbf{x}-\hat{\mathbf{x}}|\right)
$$

where $\mathbf{x}=(r, \phi)$ and $\hat{\mathbf{x}}=(\hat{r}, \hat{\phi})$. We can then find an explicit form for $P_{1}$ :

$$
P_{1}(\mathbf{x})=\frac{i}{4} \int_{\mathbb{R}^{2}} H_{0}^{(1)}\left(\sqrt{k_{\alpha}^{2}-z_{0}^{2}}|\mathbf{x}-\hat{\mathbf{x}}|\right) P_{0}(\hat{\mathbf{x}})^{3} \mathrm{~d}^{2} \hat{\mathbf{x}}
$$

Making use of both the order zero and one perturbation terms, we find the first-order perturbation theory in the cylindrical symmetry by placing $P_{0}(\mathbf{x})$ and $P_{1}(\mathbf{x})$ back into $(22)$, obtaining

$$
\begin{aligned}
& P(r, \phi)=\sum_{n=0}^{\infty} J_{n}\left(r \sqrt{k^{2}-z_{0}^{2}}\right)\left(A_{n} \sin (n \phi)+B_{n} \cos (n \phi)\right) \\
& \quad+\varepsilon \frac{i}{4} \int_{\mathbb{R}^{2}} H_{0}^{(1)}\left(\sqrt{k_{\alpha}^{2}-z_{0}^{2}}|\mathbf{x}-\hat{\mathbf{x}}|\right)\left(\sum_{n=0}^{\infty} J_{n}\left(\hat{r} \sqrt{k^{2}-z_{0}^{2}}\right)\left(A_{n} \sin (n \hat{\phi})+B_{n} \cos (n \hat{\phi})\right)\right)^{3} \mathrm{~d}^{2} \hat{\mathbf{x}} \\
& \quad+O\left(\varepsilon^{2}\right),
\end{aligned}
$$

where $\mathbf{x}=(r, \phi)$ and $\hat{\mathbf{x}}=(\hat{r}, \hat{\phi})$.

\section{The Large Mass Limit}

In this section, we will employ a method similar to the one used in [17], page 16, where we will take the mass scale of the fluid to be very large. This has the effect of modeling the superfluid as being extremely dense, by the relation $\rho_{s}=m|\Psi|^{2}$ as described in Section 1. We will start with the full, time-dependent NLS equation (5). Since we want the mass $m$ to be very large, we will take $\frac{1}{m}$ as a small parameter. Explicitly,

$$
i \hbar \Psi_{t}=-\frac{\hbar^{2}}{2} \frac{1}{m} \nabla^{2} \Psi+U\left(|\Psi|^{2}\right) \Psi+\frac{\hbar^{2}}{2 c^{2}} \frac{1}{m} \Psi_{t t},
$$

and in the limit as the mass scale $m$ tends to infinity, we obtain the equation

$$
i \hbar \Psi_{t}=U\left(|\Psi|^{2}\right) \Psi
$$


We now write $\Psi$ in the form $\Psi=R e^{i \Theta}$, where $R$ and $\Theta$ are real functions of space and time. Plugging this in to the equation above, and matching real and imaginary terms, we obtain

$$
i \hbar R_{t}=0
$$

and

$$
-\hbar \Theta_{t}=U\left(R^{2}\right)
$$

These equations have solutions

$$
R=R(\mathbf{x}) \quad \text { and } \quad \Theta(\mathbf{x}, t)=-\frac{t}{\hbar} U\left(R(\mathbf{x})^{2}\right) .
$$

Then

$$
\Psi(\mathbf{x}, t)=R(\mathbf{x}) \exp \left(-\frac{i t}{\hbar} U\left(R(\mathbf{x})^{2}\right)\right) .
$$

This is analogous to the Thomas-Fermi approximation used in the Gross-Pitaevski equation [17].

\subsection{First-Order Perturbative Solutions}

Assume we want a first-order correction in mass, say

$$
\Psi(\mathbf{x}, t)=\Psi_{0}(\mathbf{x}, t)+\frac{1}{m} \Psi_{1}(\mathbf{x}, t)+O\left(\frac{1}{m^{2}}\right) .
$$

Clearly, $\Psi_{0}(\mathbf{x}, t)$ is given by (37) above.

Expanding $U\left(|\Psi|^{2}\right)$ in the mass, we obtain

$$
U\left(|\Psi|^{2}\right)=U\left(\left|\Psi_{0}+\frac{1}{m} \Psi_{1}+O\left(\frac{1}{m^{2}}\right)\right|^{2}\right)=U\left(\left|\Psi_{0}\right|^{2}\right)+\frac{1}{m} U^{\prime}\left(\left|\Psi_{0}\right|^{2}\right)\left(\Psi_{0} \Psi_{1}^{*}+\Psi_{0}^{*} \Psi_{1}\right)+O\left(\frac{1}{m^{2}}\right),
$$

where prime indicates differentiation with respect to $|\Psi|^{2}$. At order $\frac{1}{m}$, we have

$$
i \hbar \Psi_{1, t}=-\frac{\hbar^{2}}{2} \nabla^{2} \Psi_{0}+\frac{\hbar^{2}}{2 c} \Psi_{0, t t}+U\left(\left|\Psi_{0}\right|^{2}\right) \Psi_{1}+U^{\prime}\left(\left|\Psi_{0}\right|^{2}\right) \Psi_{0}\left(\Psi_{0} \Psi_{1}^{*}+\Psi_{0}^{*} \Psi_{1}\right) .
$$

From the form of this equation, it makes sense to consider a particular solution of the form

$$
\Psi_{1}(\mathbf{x}, t)=R_{1}(\mathbf{x}) \exp \left(-\frac{i t}{\hbar} U\left(R(\mathbf{x})^{2}\right)\right)=\frac{R_{1}(\mathbf{x})}{R(\mathbf{x})} \Psi_{0}(\mathbf{x}, t) .
$$

Plugging this ansatz into equation (40), we obtain an equation for $R_{1}(\mathbf{x})$ :

$$
\frac{R_{1}}{R} i \hbar \Psi_{0, t}=-\frac{\hbar^{2}}{2} \nabla^{2} \Psi_{0}+\frac{\hbar^{2}}{2 c} \Psi_{0, t t}+U\left(\left|\Psi_{0}\right|^{2}\right) \frac{R_{1}}{R} \Psi_{0}+2 U^{\prime}\left(\left|\Psi_{0}\right|^{2}\right) \Psi_{0}\left|\Psi_{0}\right|^{2} \frac{R_{1}}{R} .
$$

From (33), we have that the left hand side cancels the third term in the right hand side. This gives us the equation

$$
-\frac{\hbar^{2}}{2} \nabla^{2} \Psi_{0}+\frac{\hbar^{2}}{2 c} \Psi_{0, t t}+2 U^{\prime}\left(\left|\Psi_{0}\right|^{2}\right) \Psi_{0}\left|\Psi_{0}\right|^{2} \frac{R_{1}}{R}
$$


Let us write

$$
J[R(\mathbf{x})]=\frac{\frac{\hbar^{2}}{2} \nabla^{2} \Psi_{0}-\frac{\hbar^{2}}{2 c} \Psi_{0, t t}}{\exp \left(-\frac{i t}{\hbar} U\left(R(\mathbf{x})^{2}\right)\right)},
$$

which is an operator involving $R(\mathbf{x}), \nabla R(\mathbf{x})$, and $\nabla^{2} R(\mathbf{x})$, as the complex exponential has been removed. We then arrive at

$$
R_{1}(\mathbf{x})=\frac{J[R(\mathbf{x})]}{2 R(\mathbf{x})^{2} U^{\prime}\left(R(\mathbf{x})^{2}\right)} .
$$

Finally, plugging this solution into (38) gives us

$$
\Psi(\mathbf{x}, t)=\left[R(\mathbf{x})+\frac{J[(R(\mathbf{x})]}{2 R(\mathbf{x})^{2} U^{\prime}\left(R(\mathbf{x})^{2}\right)} \frac{1}{m}+O\left(\frac{1}{m^{2}}\right)\right] \exp \left(-\frac{i t}{\hbar} U\left(R(\mathbf{x})^{2}\right)\right) .
$$

This gives us the first-order correction to the $m \rightarrow \infty$ limit given in (37). If we wanted to put specific conditions on this solution, we could then determine a specific form of $R(\mathbf{x})$. Still, for arbitrary large-mass solutions holding (37) as the $m \rightarrow \infty$ limit, solutions of the form (46) make complete sense.

\section{Superfluids in Curved Space-Time: A Neutron Star Metric}

In this section, we will examine a different formulation of the phenomenological Klein-Gordon model we have been using so far. We will work exclusively with $c=1$ in this section. This time, instead of modeling our superfluid on a flat spacetime, we will use a spacetime metric generally used to model the space surrounding a rotating neutron star [21]. We will use the line element for this metric introduced in [22]:

$$
\mathrm{d} s^{2}=f \mathrm{~d} t^{2}-2 n \mathrm{~d} \phi \mathrm{d} t-l \mathrm{~d} \phi^{2}-e^{\mu}\left(\mathrm{d} \rho^{2}+\mathrm{d} z^{2}\right),
$$

where $t$ is a timelike coordinate, and $\rho, \phi$ and $z$ are cylindrical parameters for the spacelike coordinates. The coefficients $l, n, \mu$ and $f$ are considered to be functions of $\rho$ and $z$, and we impose the condition

$$
f l+n^{2}=\rho^{2} .
$$

In [21] and [23], the coefficients are chosen so that this relation is made manifest. From this line element, we may calculate a metric

$$
g_{\alpha \beta}=\left(\begin{array}{cccc}
f & 0 & -n & 0 \\
0 & -e^{\mu} & 0 & 0 \\
-n & 0 & -l & 0 \\
0 & 0 & 0 & -e^{\mu}
\end{array}\right) .
$$

This gives rise to a determinant

$$
g=-\left(f l+n^{2}\right) e^{2 \mu}=-\rho^{2} e^{2 \mu}
$$

and an inverse metric

$$
g^{\alpha \beta}=\left(\begin{array}{cccc}
\frac{l}{\rho^{2}} & 0 & -\frac{n}{\rho^{2}} & 0 \\
0 & -e^{-\mu} & 0 & 0 \\
-\frac{n}{\rho^{2}} & 0 & -\frac{f}{\rho^{2}} & 0 \\
0 & 0 & 0 & -e^{-\mu}
\end{array}\right)
$$


We can plug these into the formula for the Laplacian in generalized curvilinear coordinates:

$$
\square \Phi=\frac{1}{\sqrt{|g|}} \partial_{\mu}\left(\sqrt{|g|} g^{\mu \nu} \partial_{\nu} \Phi\right)
$$

to obtain

$$
\square \Phi=\frac{l}{\rho^{2}} \Phi_{t t}-\frac{2 n}{\rho^{2}} \Phi_{t \phi}-\frac{f}{\rho^{2}} \Phi_{\phi \phi}-\frac{e^{-\mu}}{\rho} \Phi_{\rho}-e^{-\mu} \Phi_{\rho \rho}-e^{-\mu} \Phi_{z z} .
$$

Plugging this into (1), we obtain

$$
\frac{l}{\rho^{2}} \Phi_{t t}-\frac{2 n}{\rho^{2}} \Phi_{t \phi}-\frac{f}{\rho^{2}} \Phi_{\phi \phi}-\frac{e^{-\mu}}{\rho} \Phi_{\rho}-e^{-\mu} \Phi_{\rho \rho}-e^{-\mu} \Phi_{z z}+f(\Phi)=0 .
$$

Here, we see explicitly terms of the form $\Phi_{t t}$ and $\Phi_{t \phi}$, suggesting the presence of rotational forces. In fact, with the curved spacetime forms of the Coriolis and centrifugal forces given in [15]:

$$
R_{\text {coriolis }}=\frac{2 \Omega}{g_{t t}} \frac{\partial^{2}}{\partial t \partial \phi}=\frac{2 \Omega}{f} \frac{\partial^{2}}{\partial t \partial \phi},
$$

and

$$
R_{\text {centrifugal }}=\frac{\Omega^{2}}{g_{t t}} \frac{\partial^{2}}{\partial \phi^{2}}=\frac{\Omega^{2}}{f} \frac{\partial^{2}}{\partial \phi^{2}},
$$

where, per [15], the angular velocity $\Omega$ is given by $\Omega=-\frac{n}{l}$. We may then think of this spacetime metric as representing a frame rotating with angular velocity

$$
\Omega=\frac{n(\rho, z)}{l(\rho, z)} .
$$

This is a manifestation of the effect of the neutron star's rotation on surrounding spacetime.

\subsection{Determination of the Coefficients}

We would like to put equation (54) into a form that we can solve, at least numerically. The first thing we need to do is to ascertain the form of $f, n, l$, and $\mu$. As determined in [24], in order for the metric to satisfy Einstein's field equations, the coefficients must solve the following equations:

$$
\begin{gathered}
\mu_{\rho \rho}+\mu_{z z}=\frac{1}{2 \rho^{2}}\left(f_{\rho} l_{\rho}+f_{z} l_{z}+n_{\rho}^{2}+n_{\rho}^{2}\right), \\
\mu_{\rho}=-\frac{1}{2 \rho}\left(f_{\rho} l_{\rho}-f_{z} l_{z}+n_{\rho}^{2}-n_{z}^{2}\right), \\
f_{\rho \rho}-\frac{1}{\rho} f_{\rho}+f_{z z}=-\frac{f}{2 \rho^{2}}\left(f_{\rho} l_{\rho}+f_{z} l_{z}+n_{\rho}^{2}+n_{\rho}^{2}\right), \\
l_{\rho \rho}-\frac{1}{\rho} l_{\rho}+l_{z z}=-\frac{l}{2 \rho^{2}}\left(f_{\rho} l_{\rho}+f_{z} l_{z}+n_{\rho}^{2}+n_{\rho}^{2}\right), \\
n_{\rho \rho}-\frac{1}{\rho} n_{\rho}+n_{z z}=-\frac{n}{2 \rho^{2}}\left(f_{\rho} l_{\rho}+f_{z} l_{z}+n_{\rho}^{2}+n_{\rho}^{2}\right) .
\end{gathered}
$$


We would also like to put down some boundary conditions for these coefficients. We would like to consider the metric as describing an isolated neutron star at the spatial origin, and so we would like to approach flat spacetime as the distance from the origin increases. This gives rise to the following asymptotic boundary conditions as $r:=\sqrt{\rho^{2}+z^{2}} \rightarrow \infty$ :

$$
f \rightarrow 1, \quad l \rightarrow \rho^{2}, \quad n \rightarrow 0, \quad \mu \rightarrow 0 .
$$

Note that we are using a different signature here for Minkowski spacetime than in previous sections. Taking our cue from [24], we will restrict the condition on $n$ to something more specific:

$$
n \sim \frac{\lambda \rho^{2}}{r^{3}} \quad \text { as } \quad r \rightarrow \infty
$$

Physically, $\lambda$ may be said to correspond to the angular momentum of our neutron star (see [24]). Thanks to the calculations performed in [24], we may write our three coefficients in terms of a new function $\chi$ :

$$
\begin{gathered}
f=\left(\alpha \cosh \left(\chi_{z}\right)+\beta \sinh \left(\chi_{z}\right)\right)^{-1}, \\
l=\rho^{2}\left[\frac{1}{f}-\left(\alpha^{2}-\beta^{2}\right) f \chi_{\rho}^{2}\right], \\
n=\sqrt{\alpha^{2}-\beta^{2}} f \rho \chi_{\rho},
\end{gathered}
$$

and

$$
\mu_{z}+\frac{f_{z}}{f}=\rho \chi_{\rho z} \chi_{z z}
$$

where $\alpha$ and $\beta$ are arbitrary constants. Note that $\chi$ must then solve the equation

$$
\chi_{\rho \rho}+\frac{1}{\rho} \chi_{\rho}+\chi_{z z}=0
$$

Our boundary conditions for $l, n$ and $f$ as $r \rightarrow \infty$ will then give the following conditions for $\chi$ as $r \rightarrow \infty$ :

$$
\chi_{\rho} \sim \frac{\lambda^{\prime} \rho}{r^{3}}, \quad \text { and } \quad \chi_{z} \rightarrow A,
$$

where $\lambda^{\prime}=\frac{\lambda}{\sqrt{\alpha^{2}-\beta^{2}}}$, and $A$ is a constant such that $\alpha \cosh (A)+\beta \sinh (A)=1$. Our general solution is then

$$
\chi=A z-\frac{\lambda^{\prime}}{r}+\cdots
$$

where the rest of the terms are arbitrary powers of $\frac{1}{r}$. For the sake of convenience, we will set these terms to zero. Also for the sake of convenience, we will set $\beta$ to zero and $\alpha$ to one, which will also set $A$ to zero. $\chi$ and the coefficients can now be expressed in the form:

$$
\begin{gathered}
\chi=-\frac{\lambda}{r}, \\
f=\operatorname{sech}\left(\frac{\lambda z}{r^{3}}\right),
\end{gathered}
$$




$$
\begin{gathered}
l=\rho^{2}\left[\cosh \left(\frac{\lambda z}{r^{3}}\right)-\frac{\lambda^{2} \rho^{2}}{r^{6}} \operatorname{sech}\left(\frac{\lambda z}{r^{3}}\right)\right], \\
n=\frac{\lambda \rho^{2}}{r^{3}} \operatorname{sech}\left(\frac{\lambda z}{r^{3}}\right)
\end{gathered}
$$

and

$$
\mu_{z}=-\frac{3 \lambda^{2} \rho^{2} z}{r^{10}}\left(\rho^{2}-2 z^{2}\right)-\frac{f_{z}}{f}
$$

We can integrate $(75)$ to find

$$
\mu=\frac{\rho^{2} \lambda^{2}\left(\rho^{2}-8 z^{2}\right)}{8 r^{8}}-\ln \left(\operatorname{sech}\left(\frac{\lambda z}{r^{3}}\right)\right)
$$

where we have set the constant of integration to zero so that $\mu \rightarrow 0$ as $r \rightarrow \infty$. Now that we have our forms for the coefficients, we will start to get some solutions for equation (54).

\subsection{Nonlinear Schrödinger Equation and the Large Mass Limit}

Consider our equation of motion (54). We will perform the same transformation we used in flat spacetime to transform to a nonlinear Schrödinger equation. We will use the same ansatz $f(\Phi)=$ $-V^{\prime}\left(|\Phi|^{2}\right) \Phi$ as we have done in previous discussions. Here, the transformation gives us

$2 i \omega_{0} \Psi_{t}=\Psi_{t t}+\frac{2 i n \omega_{0}}{l} \Psi_{\phi}-\frac{2 n}{l} \Psi_{t \phi}-\frac{f}{l} \Psi_{\phi \phi}-\frac{\rho e^{-\mu}}{l} \Psi_{\rho}-\frac{\rho^{2} e^{-\mu}}{l}\left(\Psi_{\rho \rho}+\Psi_{z z}\right)+\left(\frac{\rho^{2}}{l} V^{\prime}\left(|\Psi|^{2}\right)-\omega_{0}^{2}\right) \Psi$.

To make this look analogous to our nonlinear Schrödinger equation in flat spacetime, we will first absorb $\omega_{0}^{2}$ into the potential by setting $U\left(|\Psi|^{2}, \rho, z\right)=\frac{\rho^{2}}{l} V^{\prime}-\omega_{0}^{2}$. Now we may make the substitution $\omega_{0}=\frac{m}{\hbar}$, where the introduced mass term $m$ is analogous to the mass $m$ that we introduced in the flat spacetime case. We then rearrange the equation to obtain

$$
i \hbar \Psi_{t}=-\frac{\hbar^{2} \rho^{2} e^{-\mu}}{2 m l}\left(\Psi_{\rho \rho}+\frac{1}{\rho} \Psi_{\rho}+\frac{f e^{\mu}}{\rho^{2}} \Psi_{\phi \phi}+\Psi_{z z}\right)-\frac{n \hbar^{2}}{2 m l} \Psi_{t \phi}+\frac{i n \hbar}{l} \Psi_{\phi}+\frac{\hbar^{2}}{2 m} \Psi_{t t}+U \Psi .
$$

Note that, in the limit $r \rightarrow \infty$, we retrieve the nonlinear Schrödinger equation we found in equation (5). As before, we want to study the solution in the large mass limit $m \rightarrow \infty$. We see that the solution in this limit must solve the equation

$$
i \hbar \Psi_{t}=\frac{i n \hbar}{l}+U \Psi
$$

We will make the substitution $\Psi=R e^{i \Theta}$, where $R(t, \mathbf{x})$ and $\Theta(t, \mathbf{x})$ are real functions that depend in general on both space and time. Substituting these in, we arrive at the equation

$$
i \hbar\left(R_{t}+i R \Theta_{t}\right)=\frac{i n \hbar}{l}\left(R_{\phi}+i R \Theta_{\phi}\right)+U\left(R^{2}, \rho, z\right) R .
$$

As before, we will begin by taking the imaginary parts of this equation:

$$
\hbar R_{t}=\frac{n \hbar}{l} R_{\phi}
$$


We can solve this with the method of characteristics to find

$$
R(\rho, \phi, z, t)=\hat{R}\left(\phi+\frac{n(\rho, z)}{l(\rho, z)} t\right)
$$

where $\hat{R}$ is an arbitrary differentiable function. We have written $n$ and $l$ with their arguments, to highlight the dependence of $R$ on all variables. We can also look at the real terms in (80) to find

$$
\Theta_{t}-\frac{n}{l} \Theta_{\phi}=-\frac{1}{\hbar} U\left(R^{2}, \rho, z\right) .
$$

Again, this can be solved using the method of characteristics. We find

$$
\Theta(\rho, \phi, z, t)=\frac{\phi l(\rho, z)}{\hbar n(\rho, z)} U\left(\hat{R}\left(\phi+\frac{n(\rho, z)}{l(\rho, z)} t\right)^{2}, \rho, z\right)+\hat{\Theta}\left(\phi+\frac{n(\rho, z)}{l(\rho, z)} t\right) .
$$

From (82) and (84), we then have a final form for our large-mass limit:

$$
\begin{aligned}
\Psi(\rho, \phi, z, t)=\hat{R} & \left(\phi+\frac{n(\rho, z)}{l(\rho, z)} t\right) \\
& \times \exp \left[i \hat{\Theta}\left(\phi+\frac{n(\rho, z)}{l(\rho, z)} t\right)+\frac{i l(\rho, z) \phi}{\hbar n(\rho, z)} U\left(\hat{R}\left(\phi+\frac{n(\rho, z)}{l(\rho, z)} t\right)^{2}, \rho, z\right)\right] .
\end{aligned}
$$

This gives us the curved-spacetime version of the Thomas-Fermi approximation, valid for rotating neutron stars under the model put forward in [24]. It should be noted that as opposed to our result in Section 3, our solution in the large-mass limit in this metric is not a stationary solution. That is, the density $\Psi$ of the fluid will change over time. We can interpret this as an effect of the rotation of the Neutron star on the dynamics of the system.

\section{Conclusions}

We have found a number of potentially interesting asymptotic solutions for a nonlinear KleinGordon (NLKG) equation which can be used as a phenomenological model for relativistic superfluid behavior [15]. We looked for stationary solutions to the NLKG equation, considering both the spherical and cylindrical cases. We found an asymptotic solution for a spherically-symmetric distribution of superfluid in flat spacetime, which turned out to have high accuracy (when compared to numerical simulations) for surprisingly large strengths in the nonlinearity. Interestingly, we saw that including a finite speed of light $(c<\infty)$ actually leads to more regular solutions, while the non-relativistic limit $(c \rightarrow \infty)$ actually gives less regular solutions. A similar but more complicated solution was provided for a cylindrical superfluid distribution.

Moving on from stationary solutions, we discussed perturbations about the large-mass limit in Section 3, and found that to first order, these solutions are stationary, meaning the density distribution of superfluid will not change over time in the large-mass limit. In order to generalize these results to curved spacetime, we considered a specific spacetime metric used to model motion in the space surrounding a rotating neutron star. We investigated the conditions imposed on this metric by Einstein's field equations, and then proceeded to find the Thomas-Fermi or large-mass 
approximation in this spacetime metric, with the surprising result that it turned out to permit non-stationary solutions, even though the corresponding approximation in Minkowski spacetime only allows for stationary states. Further research into the large-mass perturbation in the case of the neutron star metric may be useful in modeling the superconductivity of the interior of neutron stars [15]. Indeed, our results suggest that a variety of solutions can be possible for this problem, not just the stationary states which are expected in the flat spacetime regime.

\section{References}

[1] D. Langlois, D. M. Sedrakian, and B. Carter, Differential rotation of relativistic superfluid in neutron stars Monthly Notices of the Royal Astronomical Society 297(4) (1998) 1189-1201.

[2] J. A. Sauls, Superfluidity in the Interiors of Neutron Stars, Timing Neutron Stars. Springer, Netherlands (1989), pp. 457-490.

[3] A. D. Sedrakian and D. M. Sedrakian, Superfluid core rotation in pulsars. I. Vortex cluster dynamics, The Astrophysical Journal 447 (1995) 305.

[4] S. Bonazzola, E. Gourgoulhon, M. Salgado, and J. A. Marck, Axisymmetric rotating relativistic bodies: A new numerical approach for 'exact' solutions, Astronomy and Astrophysics 278 (1993) 421-443.

[5] M. Salgado, S. Bonazzola, E. Gourgoulhon, and P. Haensel, High precision rotating netron star models 1: Analysis of neutron star properties, Astronomy and Astrophysics 291 (1994) $155-170$.

[6] G. L. Comer, and D. Langlois, Hamiltonian formulation for relativistic superfluids, Classical and Quantum Gravity 11 (1994) 709.

[7] R. L. Davis, Relativistic superfluids and vortex rings, Physical Review D 40(12) (1989) 4033.

[8] U. Ben-Ya'acov, Strings, superfluid vortices, and relativity, Physical Review D 44(8) (1991) 2452 .

[9] R. L. Davis and E. P. S. Shellard, Global strings and superfluid vortices, Physical Review Letters 63(19) (1989) 2021.

[10] V. Keränen, E. Keski-Vakkuri, S. Nowling, and K. P. Yogendran, Inhomogeneous structures in holographic superfluids. II. Vortices, Physical Review D 81(12) (2010) 126012.

[11] B. Carter and D. Langlois, Relativistic models for superconducting-superfluid mixtures, Nuclear Physics B 531 (1998) 478-504.

[12] K. W. Schwarz, Three-Dimensional Vortex Dynamics in Superfluid He 4: Line-line and Lineboundary Interactions, Physical Review B 31(9) (1985) 5782.

[13] H. Hasimoto, A Soliton on a Vortex Filament, Journal of Fluid Mechanics 51(3) (1972) 477485. 
[14] R. A. Van Gorder, Quantum Hasimoto Transformation and Nonlinear Waves on a Superfluid Vortex Filament under the Quantum Local Induction Approximation, Physical Review E 91(5) (2015) 053201.

[15] C. Xiong, M. R. R. Good, Y. Guo, X. Liu, and K. Huang, Relativistic Superfluidity and Vorticity from the Nonlinear Klein-Gordon Equation, Physical Review D 90(12) (2014) 125019.

[16] V. L. Ginzburg and L. D. Landau, On the theory of superconductivity, Zh. eksp. teor. Fiz 20 (1950) 1064-1082.

[17] K. Mallory and R. A. Van Gorder, Stationary Solutions for the Nonlinear Schrödinger Equation Modeling Three-Dimensional Spherical Bose-Einstein Condensates in General Potentials, Physical Review E 92(1) (2015) 013201.

[18] G. Evequoz and T. Weth, Real Solutions to the Nonlinear Helmholtz Equation with Local Nonlinearity, Archive for Rational Mechanics and Analysis 211(2) (2013) 359-388.

[19] R. T. Couto, Green's functions for the wave, Helmholtz and Poisson equations in a twodimensional boundless domain, Revista Brasileira de Ensino de Física 35(1) (2013) 1304.

[20] F. B. Hildebrand, Advanced Calculus for Applications Prentice-Hall, 1976

[21] G. Pappas and A. Apostolatos, An all-purpose metric for the exterior of any kind of rotating neutron star, Monthly Notices of the Royal Astronomical Society 429(4) (2013) 3007-3024.

[22] T. Lewis, Some Special Solutions of the Equations of Axially Symmetric Gravitational Fields, Proceedings of the Royal Society A 136(829) (1932) 176-192.

[23] V. S. Manko and E. Rulz, Exterior field of slowly and rapidly rotating neutron stars: Rehabilitating spacetime metrics involving hyperextreme objects, Physical Review D 93(10) (2016) 104051.

[24] A. Papapetrou, Eine rotationssymmetrische Lösung in der allgemeinen Relativitätstheorie, Annalen der Physik 447(4-6) (1953) 309-315. 